\title{
INTRAOSSEOUS ACCESS — FUTURE, PRESENT AND EVERYDAY LIFE
}

\author{
Agata Dabrowska ${ }^{1,2}$, Marek Dabrowski $^{1,2}$, Karol Bielski ${ }^{3}$, Adrian Maciejewski ${ }^{1,4}$, Emilia Surzyn ${ }^{4}$ \\ ${ }^{1}$ Department of Rescue and Disaster Medicine, Poznan University of Medical Sciences, Poland \\ ${ }^{2}$ Polish Society of Medical Simulation, Poland \\ ${ }^{3}$ MEDITRANS The Voivodeship Emergency Medical Service and Sanitary Transport, Warsaw, Poland \\ ${ }^{4}$ Journal Club, Poznan University of Medical Sciences, Poland
}

\begin{abstract}
Today, intraosseous access (IO) is not only an alternative method of administration of pharmacotherapy or fluids; it is often used in life-threatening conditions. Although previously, it was a method commonly used in paediatrics or in the military, for several years it has been advocated as the primary access point for patients in a critical condition. While this applies mainly to children, it may also include adults in a hospital setting, as well as in the emergency department. Oftentimes it is used when intravenous access is difficult or the patient is seriously ill. Many scientific circles at the American Heart Association (AHA) and European Resuscitation Council (ERC) approve this method.
\end{abstract}

KEY WORDS: intraosseous access (IO), intravenous access (IV), life-threatening condition, BIG, FAST, NIO Disaster Emerg Med J 2017; (2)1: 19-26

\section{INTRODUCTION}

The commencement of administering intraosseous drugs dates back to the early years of the twentieth century [1-4]. Today, we know that if intravenous access is difficult or impossible, intraosseous access should be considered. Currently, it also is a very effective method of administering drugs and fluids in adults. The concentration of drug administered intraosseously in most situations is comparable to the amount seen in the plasma when giving the drug intravenously (IV). Therefore, in recent years there has been an increased availability of mechanical access to 10 , resulting in its increased use. Today, intraosseous access is available to both adults and children (including infants) with a variety of 10 access points such as the sternum, the humerus, as well as both the proximal and distal portions of the tibia and radius [2]. Gaining access to the circulatory system is one of the most important tasks in patients suffering from sudden cardiac arrest. It is not easy to get a venous line in patients who, within the first two minutes of CPR, have failed to receive proper access. In such situations, accessing the bone marrow should be considered as the best alternative [5]. The choice and decision to use a particular device depends on the local protocol, as well as the skills and knowledge of the available equipment by the medical personnel. Often, however, the reality is that intraosseous devices are available but are not being used [3]. Of course, if possible, drugs in life-threatening conditions should be administered intravenously. However, always when complications arise, the immediate appropriate measure should be intraosseous [1]. Intraosseous access is obtained by injecting a needle directly into the marrow cavity using a specially designed device. This is an optional way to supply medication and fluids when there are IV complications or when it is simply impossible to access collapsed peripheral veins. The time it takes to introduce the needle in order to ensure contact with the marrow cavity is under a minute. With a properly placed intraosseous device, the infusion site can flow to $125 \mathrm{ml} / \mathrm{min}$. In addition, it makes it possible to access material for laboratory tests such as blood type, 
gas analysis, morphology, haemoglobin, and electrolyte levels $[6,7]$. The contraindications in establishing intraosseous access are fractures or other injuries at the proposed injection site, as well as local infection in the affected area. There is always a risk of complications that may arise when introducing an intraosseous injection. The most common are hematoma, inflammation, and bone fracture. Through the use of $\mathrm{IO}$, bone marrow can be aspirated to check blood type and analyse the measurements of blood gases (gas levels are comparable to the blood gas levels in the central veins, provided intraosseous drugs were not earlier administered) [7]. Intraosseous access should be maintained only until a stable access to the venous vasculature is available. Ideally, needles should be removed in less than 24 hours $[8,9]$.

This paper is a literature meta-analysis of the last 10 years in the context of access to the vascular bed. The aim of the analysis is to describe the use of equipment, actions related to intraosseous access and its constraints. The data collected is divided into the following categories: indications, benefits and contraindications; technical rules; care during access; and possible complications. The collected literature on intraosseous vascular access supports intraosseous injection as the main alternative to vascular access. The collected literature further supports how the connection and supply of drugs and fluids is quick and easy through the use of intraosseous vascular access.

\section{General rules for accessing the vasculature}

The use of intraosseous injections should follow the same rules as injections using intravenous access. One should, however, emphasize adherence to the following key points in order to perform the procedure.

1. Sterilization of the skin at the access site;

2. Maintaining manual stabilization of the limbs (bones) during injection;

3. Aspiration directly after the introduction of the needle to confirm the correct positioning;

4. For conscious patients prior to administering medication or fluids, one should administer a local anaesthetic (preferably lidocaine) at the intraosseous site in order to reduce the pain during subsequent infusions according to the principle: 2 of 2 for 2 times ( 2 of 2 for $2 \times 2$ ). This means: $2 \mathrm{ml}$ of $2 \%$ lidocaine for 2 minutes 2 times;

5. Confirmation of proper access to the bone marrow by administration of $10 \mathrm{ml}$ of fluid after administration of the drugs;
6. Patient records should state the execution of the intraosseous procedure;

7. Evaluation of the 10 site for signs of extravasation.

\section{Indications}

According to the regulations, if intravenous access is difficult or impossible, one should always consider intraosseous access. This means that with each life-threatening condition, when there is no possibility of intravenous access, there is an obligation to seek an alternative route, or intraosseous access. The absolute indications include:

1. Paediatric patients in a critical condition;

2. Cardiac arrest in children and adults;

3. Failure to obtain intravenous access after two attempts (e.g. in victims with hypovolemia, in whom rapid intravenous access cannot be gained), or no possibility of the rapid establishment of peripheral intravenous access [10-11].

\section{Contraindications}

1. Symptoms of infection of soft tissue at the injection site;

2. Broken bones;

3. Compartment Syndrome;

4. Limb injury with damage to the vascular bed;

5. A previous attempt to establish intraosseous access within 24 hours;

6. Relative contraindications include: difficulty in identifying the anatomical landmarks, in these patients. Moreover, IO access should be used with caution, as this may cause damage to nearby structures $[1-3,5,12]$.

\section{Complications associated with obtaining intraosseous (I0) access:}

1. Bruising;

2. Infection at the injection site;

3. Pain at the injection site;

4. Broken bones (mainly in newborns and patients with osteoporosis);

5. Osteomyelitis;

6. Sepsis;

7. Fat embolism;

8. Bone fracture, and splintering if the needle is too thick;

9. Inflammation at the site of the bone marrow cavity;

10.Bone marrow aplasia (destruction of bone marrow stromal connective tissue); 
11. Cardiac tamponade;

12. Extravasation of subcutaneous drugs;

13. Drug leakage from injection site;

14. Hypertension or pressure in the fascial space [10-12]

\section{Conditions associated with executing inappropriate access}

1. Puncture outside of the designated area;

2. Bone puncture;

3. Fluid transfused into the yellow marrow cavity of the bone marrow.

\section{Access sites}

Despite having a limited number of places where it is possible to achieve an access site, placement of a device depends essentially on the type of equipment used. Some devices are limited to specific locations. The manubrium should be used primarily in military conditions. A bulletproof vest generally protects the chest; consequently it is subject to a decreased number of fractures. The upper and lower limbs are the places that are the most vulnerable to high-energy phenomena leading to common injuries such as fractures and/or massive haemorrhage as a result of gunshot wounds, explosions leading to shrapnel lodging in the skin, amputations, defragmentation tissue, all of which form contraindications to establishing 10 access. Outlined below are suitable places for 10 access:

1. Tibial Bone:

a) Proximal part of the tibia - locate the tuberosity of the tibia:

- Adults - $2 \mathrm{~cm}$, medially and $1 \mathrm{~cm}$ cranially from the tibial tuberosity (Fig. 1);

- Paediatrics - 1-2 cm medially and around $1 \mathrm{~cm}$ towards the foot from the tibial tuberosity to the flat portion of the bone;

— Older children — slightly lower injection site than in paediatric patients;

b) Part of the distal tibia - locate the medial malleolus:

- Adults - insertion site is located the width of two fingers above the upper edge of the ankle; $1-2 \mathrm{~cm}$ proximal to the base of the medial malleolus $(4-5 \mathrm{~cm}$ above the end of the medial malleolus);

- Paediatric patients - insertion site is located one finger width above the upper edge of the ankle.

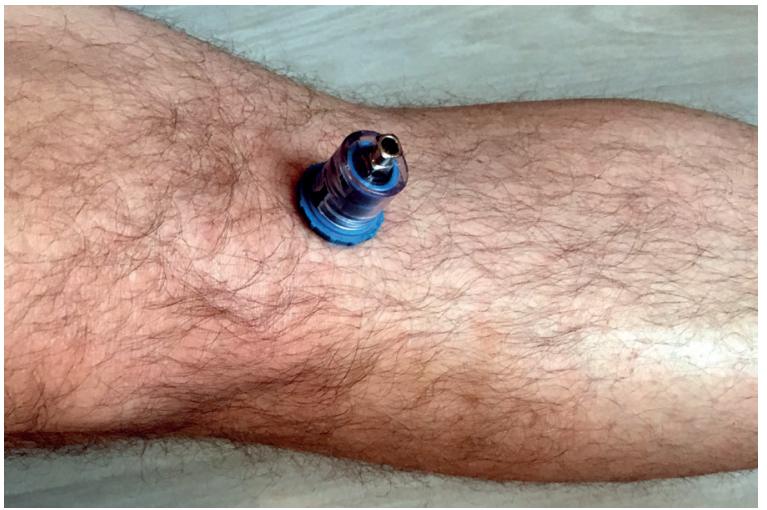

FIGURE 1. Intraosseous access in location of Tibial Bone

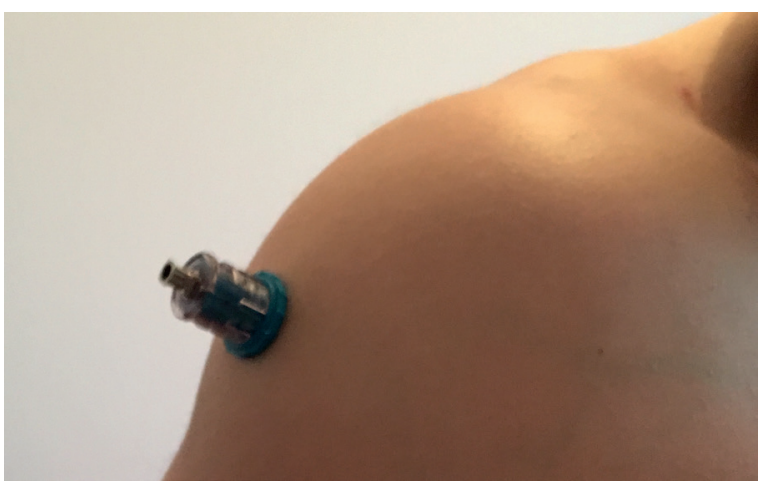

FIGURE 2. Intraosseous access in location of Humerus

\section{Humerus:}

It is recommended that the patient be in a sitting position. The upper limb which will enable 10 access should be bent at the elbow, in order that the hand is touching the navel. One should then locate the core of the humerus, moving toward the shoulder. Near the shoulder one should find a protruding bone fragment - namely, the greater tubercle of the humerus. Using one's other hand, one should feel for the protruding portion of the humerus in order to mark the injection site for 10 access. In small children, finding the greater tuberosity of the humerus may be difficult (Fig. 2).

\section{Manubrium:}

Locate the manubrium, approximately $2 \mathrm{~cm}$ below the sternal notch, and insert at 90 degrees to the skin. The fluid flows into the internal mammary veins, then into the azygous veins, and finally into the central circulation [13-15].

\section{EQUIPMENT FOR INTRAOSSEOUS ACCESS}

Regarding the access and type of mechanism used for the injection, devices can be subdivided into three major types. 


\section{Manual Devices (manual trocar)}

Requiring the use of large forces, manual devices are commonly used in hospital care and not used in emergency care in a pre-hospital setting. They are recommended for children in order to access the lower parts of the lower limbs. Although their ease of use and low cost are an advantage, the use of excessive force in relation to other devices is a drawback.

\section{Jamshidi Needle}

This device is limited to paediatric use on lower limb sites as excessive force is needed for insertion in adult patients (Fig. 3). These disposable intraosseous infusion needles provide one with a safe and simple technique in order to gain vascular access for delivering fluids and drugs to critically ill or injured children. They feature a sharp lancet point to facilitate entry into the bone and Luer slip / Luer lock connection for a secure fit to any syringe. An adjustable guard helps one control the depth of needle insertion while a twist-off cap holds the stylet securely in place. Intraosseous infusion is recommended only in life-threatening emergencies where vascular access is critical and conventional forms of access are not available or unobtainable. There are 2 sizes: 15 gauge for those 9 months or older and 18 gauge for those younger than 9 months [16].

\section{Spring Devices (such as a gun)}

The spring devices are used to aid penetration into the bone marrow. These are disposable devices used to introduce needles into the lower limb (tibia) or the upper lower limb (radius) and allow access to the sternum. These systems require training to ensure their proper use and inspect techniques.

\section{BIG — Bone Injection Gun}

The world's first automatic, user-friendly 10 device brings one safe, rapid 10 access for the delivery of fluids and medications at flow rates similar to peripheral IV infusion (Fig. 4). With the help of a Bone Injection Gun, IO infusion can be established within seconds. The B.I.G. presents an easy and safe "position and press" mechanism. These are licensed for use in the proximal tibia and the humerus. The device is commonly colour coded; blue for adults, red for children and green for use by veterinary surgeons. Typically used at the upper tibia plateau, the technique is simple to learn through brief training. The BIG is rapidly becoming the device of choice for physicians and paramedics in emergency medical

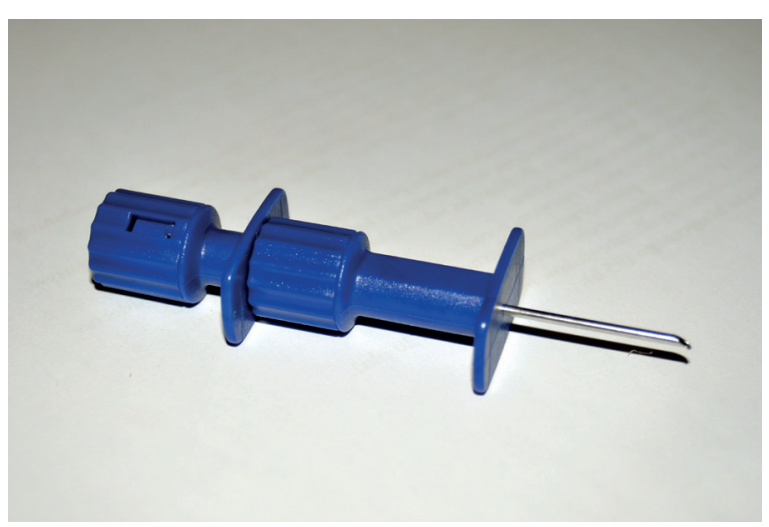

FIGURE 3. Jamshidi intraosseous needle

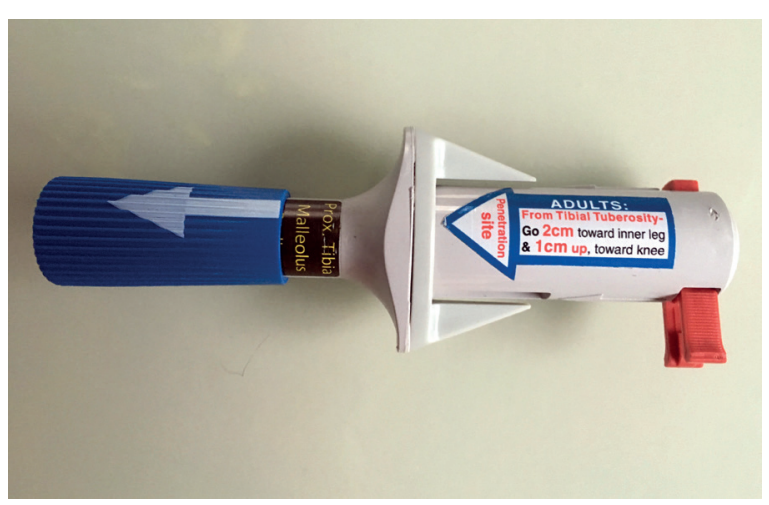

FIGURE 4. BIG — Bone Injection Gun

services, hospitals, armed forces, government agencies, and tactical environments [17].

\section{NIO Adult - New Intraosseous Device}

The NIO is an automatic intraosseous device packaged for safe, quick, and easy vascular access (Fig. 5). It is a simple, automatic intraosseous device ready to use for safe, quick, and easy vascular access. There are no drills, no batteries, nor extra parts. The NIO is small, light, and easy to use in even the most demanding situations. It is designed for easy injection into either the tibia or the humerus in all clinical settings and is intended for use on adults/those twelve years of age or older. The NIO is for use on the proximal tibia or the humeral head, and proved to be safe regarding the bone - resulting in zero incidence of bone fracture as observed by post-insertion X-rays. Its features include the following:

1. Sterile - zero chance of cross-contamination;

2. Vascular access in as few as 10 seconds;

3. Disposable and single-use;

4. No external power source or battery needed;

5. Pocket-sized and lightweight - $3.5 \mathrm{oz}$.;

6. No maintenance required;

7. Patient-friendly, no exposed needle; 


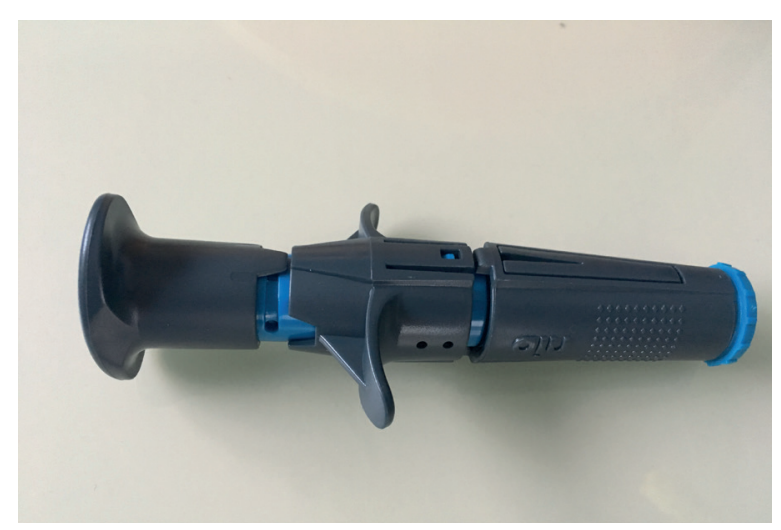

FIGURE 5. NIO Adult — New Intraosseous Device

8. Assembled and ready to use in a package;

9. A 5-year shelf life;

10.2 sizes - adult and paediatric [18].

\section{FAST1 Sternal I0}

As a highly effective alternative to traditional peripheral intravenous access, FAST1 gives one the critical ability to quickly establish vascular access, as well as rapidly and easily deliver emergency fluids and medications into the vascular system through the bone marrow of the manubrium. Safe to use in patients 12 years of age and older, FAST1 can be effectively used during CPR and when a cervical spine collar is in place.

Reasons to choose FAST1 include the following:

1. FAST1 is always ready to use and does not require batteries;

2. As the sternum is easier to locate than long bones, the speed and ease of finding the access site is increased;

3. It provides reliable and consistent access with automatic depth adjustment;

4. Easy to learn and difficult to make mistakes, even for novice users;

5. The injection site and the manner of its establishment and location makes it safe and difficult to remove in unwanted circumstances, e.g. during transport;

6. It provides less pain for the patient both during insertion and the administration of drugs. In most cases, lidocaine is not required;

7. It consists of a compact and lightweight design.

\section{The benefits of the FAST1}

The FAST1 allows for the use of any fluid or medication that can be injected intravenously. The American Heart Association, Advanced Trauma Life Support and the
European Resuscitation Council have approved it in the care and treatment of critically ill patients. The FAST IO 1 is also used and is dedicated for military medicine and Tactical Combat Casualty Care guidelines.

The FAST1 can be placed and vascular access (essentially equivalent to a central venous line) achieved in 10 seconds with medications reaching the heart within 30 seconds. Its features include the following: 1. It provides the quickest route to the heart of any IO access device and peripheral IV;

2. Exact placement is achieved every time;

3. Automatic depth control is provided for safe delivery above the lungs and heart;

4. It is easy to use, even for new medical professionals;

5. It is a sterile, single-use, disposable product with no risk of cross-contamination;

6. It is flexible with soft, low-profile, secure tubing;

7. Its lightweight design allows its portability in medic packs [19-21].

\section{Drill-based system (drill)}

These devices are easy to use, requiring less training. Battery-powered drills enable a broader range of access points to the injection site. They consist of a reusable drill and disposable needles.

\section{EZ-IO — Easy Intraosseous}

This is a handheld drill device, which consists of a power driver and needle set (Fig. 6). There are three different needle and stylet combinations, suited to different patient sizes. There are needles for patients weighing 3-39 kg, more than $40 \mathrm{~kg}$, and a large needle for patients with excess soft tissue. Its features include the following:

1. The EZ-IO Intraosseous Vascular Access System is a fast vascular access option that provides peripheral venous access with central venous

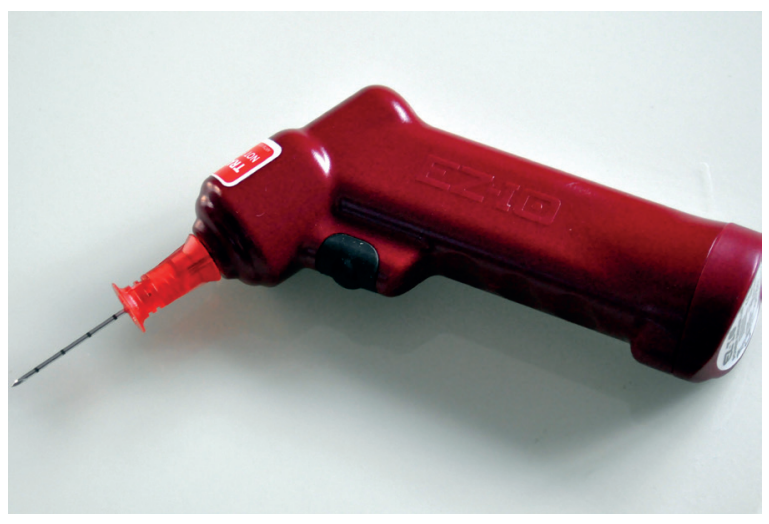

FIGURE 6. EZ-IO — Easy Intraosseous access device 
catheter performance, avoiding unnecessary central line placement and, thus, the risk of central line infection;

2. The EZ-IO Intraosseous Vascular Access System uses the intraosseous space of the humerus, tibia, and distal femur (paediatrics) as a large, non-collapsible vein;

3. The EZ-IO needle has a uniquely designed needle tip that drills a hole in the bone the same size as the needle, minimizing the risk of extravasation or dislodgement;

4. Insertion is gentle, fast and relatively painless, and is particularly beneficial for patients who require multiple IV sticks to obtain vascular access for medication [22].

According to the manufacturers, each of the methods employed through these devices provide quick access and make it possible to get the flow to $125 \mathrm{ml} / \mathrm{min}$. The same drugs given intravenously can be safely given through intraosseous insertion. To promote high flow values with fluid therapy after using intraosseous injection, a cuff is used in instances of quick transfusion. It is possible to obtain a rate greater than $125 \mathrm{ml} / \mathrm{min}$, which provides a chance to increase injection transfusions of up to $1,000 \mathrm{ml}$ in 4 minutes $(2 \times 125 \mathrm{ml}=250 \mathrm{ml}=1000 \mathrm{ml}$ over $4 \mathrm{~min})$.

\section{DISCUSSION}

A study done on Transported Helicopter Emergency Medical teams assessed that from 780 patients, intraosseous access was used 40 times, meaning in $5.1 \%$ of cases. The device used was the BIG whose efficacy in children (age $<16$ years) was $71 \%$ $(10 / 14)$ and $73 \%$ in adults (19/26). In this study no serious complications were noted [23].

In a prospective study conducted by Gazin et al., lasting one year and with prehospital staff accessing vascular entry 4,666 times, intraosseous access was performed 39 times, which accounted for only $0.84 \%$ of the total number of injections. Of these 39 injections, 30 included patients in cardiac arrest while 9 were performed in patients with preserved perfusion. The effectiveness of forming an access on the first attempt was $84 \%$ [24].

In a study by Schwartz et al., of 189 cases of EMS employees employing 10 in a prehospital setting over a period of 4 years, as much as 56\% were related to cardiovascular morbidity. Moreover, $12 \%$ were trauma-related, $11 \%$ respiratory-related and the rest, which made up to $21 \%$ of patients [25].
Leidel et al. performed a study where two intraosseous devices were compared namely, the BIG and EZ-IO. The difference in the use of both devices was found not to be statistically significant and no significant complications were observed. Both devices were used and compared in 20 trials. The efficacy of the use of the BIG reached $80 \%$ while that of the EZ-IO was 90\% [26].

In an interventional, randomized, crossover, single-centre cadaver study, a semi-automatic springload driven NIO access device was investigated. In total, 84 paramedics with less than 5 years of experience in the Emergency Medical Services participated in the study. The trial was performed on 42 adult cadavers. In each cadaver, $2 \mathrm{IO}$ accesses to the humerus head, and 210 accesses to the proximal tibia were obtained. The success rate of the first 10 attempt was $89.3 \%$ (75/84) for tibial access, and $73.8 \%$ for humeral access. Tibial $1 \mathrm{O}$ access is easier and faster to put in place than humeral $1 \mathrm{O}$ access. Humeral $I \mathrm{O}$ access may be an alternative method to tibial 10 access [27].

In a study comparing intraosseous devices based on the time needed to obtain proper access to the marrow, the BIG, FAST 1 and Jamshidi Needle were compared. The quickest method proved to be the method involving the manual trocar (Manual trocar — Jamshidi Needle) which achieved an average access time of 38 seconds, the next being the BIG and the FAST with 49 seconds and 62 seconds, respectively. The efficacy of the total devices used was $80 \%$ in adults and in children $86 \%$. No significant difference was observed in their effectiveness, nor in the number of complications [28].

\section{CONCLUSION}

In recent years, there have been a number of devices that have come on the market providing quick and easy intraosseous access. This ensures comfort for medical personnel conducting the procedure and the ability and reliability of the administration of pharmacotherapy in life-threatening conditions, especially in children and patients during cardiac arrest. For two-person medical rescue teams, this is a key way of cutting down the time needed to gain access to the vascular bed and to provide patients with analgesics and life-support pharmacotherapy. In conjunction with a tourniquet, 10 access enables rapid fluid replacement. Another advantage of intraosseous access is the ability to perform basic tests for blood gases and to determine blood type. 
However, their main disadvantage is the high costs of the intraosseous needles and sets. 10 access is an alternative to venous punctures provided by appropriately trained medical personnel who require regular training in order to maintain the skills associated with obtaining appropriate access and the ability to provide pharmacotherapy. Intraosseous access, as a form of contact with the cardiovascular system, was officially recognized by the AHA and ERC in 2010 as an alternative to intraventricular (IV) access and is regarded as providing safe and effective access to the vasculature. Complications are relatively rare, and it is important that it can be successfully used in virtually all age groups and as a tool to administer any type of pharmacotherapy that is used to access the vasculature and effectively treat patients in hypovolemic shock with fluids.

\section{Conflict of interest: None declared.}

\section{REFERENCES}

1. Link MS, Berkow LC, Kudenchuk PJ, et al. Part 7: Adult Advanced Cardiovascular Life Support: 2015 American Heart Association Guidelines Update for Cardiopulmonary Resuscitation and Emergency Cardiovascular Care. Circulation. 2015; 132(18 Suppl 2): S444-S464, doi: 10.1161/CIR.0000000000000261, indexed in Pubmed: 26472995.

2. Soar J, Nolan JP, Böttiger BW, et al. Adult advanced life support section Collaborators. European Resuscitation Council Guidelines for Resuscitation 2015: Section 3. Adult advanced life support. Resuscitation. 2015; 95: 100-147, doi: 10.1016/j.resuscitation.2015.07.016, indexed in Pubmed: 26477701.

3. Deakin CD, Nolan JP, Soar J, et al. European Resuscitation Council Guidelines for Resuscitation 2010 Section 4. Adult advanced life support. Resuscitation. 2010; 81(10): 1305-1352, doi: 10.1016/j. resuscitation.2010.08.017, indexed in Pubmed: 20956049.

4. Drinker CK, Drinker KR, Lund CC. The circulation of the mammalian bone marrow. Am J Physiol. 1922; 62: 1-92.

5. Isayama K, Nakatani T, Tsuda M, et al. Current status of establishing a venous line in CPA patients by Emergency Life-Saving Technicians in the prehospital setting in Japan and a proposal for intraosseous infusion. Int J Emerg Med. 2012; 5(1): 2, doi: 10.1186/1865-1380-5-2, indexed in Pubmed: 22230330.

6. Day MW. Intraosseous devices for intravascular access in adult trauma patients. Crit Care Nurse. 2011; 31(2): 76-89, doi: 10.4037/ ccn2011615, indexed in Pubmed: 21459867.

7. Maconochie IK, Bingham R, Eich C, et al. Paediatric life support section Collaborators. European Resuscitation Council Guidelines for Resuscitation 2015: Section 6. Paediatric life support. Resuscitation. 2015; 95: 223-248, doi: 10.1016/j.resuscitation.2015.07.028, indexed in Pubmed: 26477414.
8. Resuscitation Council (UK) (2011) Provider Manual for use in the UK: European Paediatric Life Support Course (3rd Edition). London, BMJ. www.resus.org.uk.

9. Day MW. Intraosseous devices for intravascular access in adult trauma patients. Crit Care Nurse. 2011; 31(2): 76-89, doi: 10.4037/ ccn2011615, indexed in Pubmed: 21459867.

10. Buck ML, Wiggins BS, Sesler JM. Intraosseous drug administration in children and adults during cardiopulmonary resuscitation. Ann Pharmacother. 2007; 41(10): 1679-1686, doi: 10.1345/aph.1K168, indexed in Pubmed: 17698894.

11. Kovar J, Gillum L. Alternate route: the humerus bone - a viable option for 10 access. JEMS. 2010; 35(8): 52-59, doi: 10.1016/S01972510(10)70207-2, indexed in Pubmed: 20708143.

12. Tocantins LM. Rapid Absorption of Substances Injected into the Bone Marrow. Experimental Biology and Medicine. 1940; 45(1): 292-296, doi: 10.3181/00379727-45-11657.

13. Dubick MA, Holcomb JB. A review of intraosseous vascular access: current status and military application. Mil Med. 2000; 165(7): 552-559, indexed in Pubmed: 10920658.

14. Frascone $R$, Dries $D$, Gisch $T$, et al. Obtaining vascular access: is there a place for the sternal I0? Air Med J. 2001; 20(6): 20-22, indexed in Pubmed: 11692134.

15. Porth CM. Structure and function of the musculoskeletal system. In: Porth CM. ed. Pathophysiology: Concepts of Altered Health States. 7th ed. Lippincott Williams \&Wilkins, Philadelphia, PA 2005: 1357-1366.

16. www.boundtree.com/jamshidi-intraosseous-io-needles-group (March 2017).

17. www.ps-med.com/products/vascular/BIG (March 2017).

18. www. ps-med.com/products/vascular/NIO (March 2017).

19. http://www.pyng.com/fast1 (March 2017).

20. Wagner MB, McCabe JB. A comparison of four techniques to establish intraosseous infusion. Pediatr Emerg Care. 1988; 4(2): 87-91, indexed in Pubmed: 3380751.

21. Day MW. Intraosseous devices for intravascular access in adult trauma patients. Crit Care Nurse. 2011; 31(2): 76-89, doi: 10.4037/ ccn2011615, indexed in Pubmed: 21459867.

22. www.arrowezio.com/ezio (March 2017).

23. Gerritse BM, Scheffer GJ, Draaisma JM. Prehospital intraosseus access with the bone injection gun by a helicopter-transported emergency medical team. J Trauma. 2009; 66(6): 1739-1741, doi: 10.1097/ TA.0b013e3181a3930b, indexed in Pubmed: 19509638.

24. Gazin N, Auger H, Jabre P, et al. Efficacy and safety of the EZ-IO ${ }^{\mathrm{TM}}$ intraosseous device: Out-of-hospital implementation of a management algorithm for difficult vascular access. Resuscitation. 2011; 82(1): 126-129, doi: 10.1016/j.resuscitation.2010.09.008, indexed in Pubmed: 20947238.

25. Schwartz D, Amir L, Dichter R, et al. The use of a powered device for intraosseous drug and fluid administration in a national EMS: a 4-year experience. J Trauma. 2008; 64(3): 650-655, doi: 10.1097/ TA.0b013e31814db2a6, indexed in Pubmed: 18332804. 
26. Leidel BA, Kirchhoff $C$, Braunstein $V$, et al. Comparison of two intraosseous access devices in adult patients under resuscitation in the emergency department: A prospective, randomized study. Resuscitation. 2010; 81(8): 994-999, doi: 10.1016/j.resuscitation.2010.03.038, indexed in Pubmed: 20434823.

27. Szarpak L, Truszewski Z, Smereka J, et al. A Randomized Cadaver Study Comparing First-Attempt Success Between Tibial and Humeral
Intraosseous Insertions Using NIO Device by Paramedics: A Preliminary Investigation. Medicine (Baltimore). 2016; 95(20): e3724, doi: 10.1097/MD.0000000000003724, indexed in Pubmed: 27196493.

28. Hartholt KA, van Lieshout EMM, Thies WC, et al. Intraosseous devices: a randomized controlled trial comparing three intraosseous devices. Prehosp Emerg Care. 2010; 14(1): 6-13, doi: 10.3109/10903120903349861, indexed in Pubmed: 19947861. 